\title{
Research Paper \\ Factors Predicting Daily and Instrumental Living Activities in Obese and Over- weight Patients After Coronary Artery Bypass Graft Surgery
}

\author{
Azzate Paryad ${ }^{1,2}\left(\mathbb{D}\right.$, Alireza Balaafkandah ${ }^{1}\left(\mathbb{C},{ }^{*}\right.$ Atefeh Ghanbari $^{1}$ (i)
}

1. Department of Nursing, School of Nursing and Midwifery, Guilan University of Medical Sciences, Rasht, Iran

2. GI cancer Screening \& Prevention Research Center, Guilan University of Medical Sciences, Rasht, Iran.

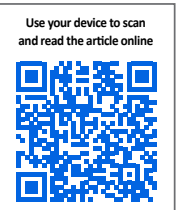

Citat ion Paryad A, Balaafkandah A, Ghanbari A. [Factors Predicting Daily and Instrumental Living Activities in Obese and Overweight Patients After Coronary Artery Bypass Graft Surgery (Persian)]. Quarterly of "The Horizon of Medical Sciences". 2020; 26(1):2-13. https://doi.org/10.32598/hms.26.1.2959.1

doi) $h$ https://doi.org/10.32598/hms.26.1.2959.1

Key words:

Activities of Daily Living, Coronary artery bypass graft, Body mass index, Instrumental Activities of Daily Living

\section{ABSTRACT}

Aims Obese or overweight people are more likely to have complications after Coronary Artery Bypass Graft (CABG) surgery. It affects the patients' postoperative abilities to perform Activities of Daily Living (ADLs) and Instrumental Activities of Daily Living (IADLs). The aim of this study was to determine the factors predicting ADLs and IADLs in overweight and obese patients after CABG.

Methods \& Materials This analytical study with cross-sectional design was conducted over a period of 4 months on 84 patients one month after CABG, who were selected using a convenience sampling method. Data were collected using a sociodemographic form, Charlson comorbidity index, Katz Index, and Lawton IADL Scale. The collected data were analyzed in SPSS software using descriptive and inferential statistics. Findings The majority of obese and overweight patients (94\%), one month after surgery, were independent of their ADLs, while most of them were in need of help in their IADLs (88.1\%). Logistic regression model showed that the variables of male gender $(P=0.004, O R=10.51, \mathrm{Cl} 95 \%=2.15-15.41)$ and carotid artery involvement $(\mathrm{P}=0.01, \mathrm{OR}=0.12, \mathrm{Cl} 95 \%=0.02-0.64)$ were the predictors of $\mathrm{ADLs}$ and IADLs in these patients.

Conclusion Obese and overweight people have impairments in performing IADLs. It is important to pay attention to the predictors including male gender and carotid artery involvement in their return to normal life after CABG.

\section{Extended Abstract}

\section{Introduction}

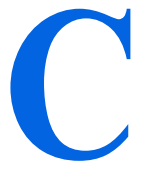

oronary Heart Disease (CHD), also called coronary artery disease, has become widespread due to lifestyle changes. In addition to causing care and economic costs, this disease is also one of the most important causes of disability [1]. The number of Coronary Artery
Bypass Graft (CABG) surgeries in the United States is estimated at 213700 per year [2]. In Iran, 16,000 to 18,000 people undergo CABG surgery $[3,4]$. There are several under lying causes of this disease. One is the overweight and obesity [5, 6], leading to an increase in the cost of surgery, damage to the healthcare system [7], and an increase in the length of hospital stay in the cardiac surgery intensive care unit (ICU) and other wards [8,9]. Obviously, patients' ability to perform daily activities after heart surgery is reduced, and obesity and overweight may increase

* Corresponding Author:

Atefeh Ghanbari, PhD.

Address: Department of Nursing, School of Nursing and Midwifery, Guilan University of Medical Sciences, Rasht, Iran.

Tel: +98 (911) 1351247

E-mail: at_ghanbari@gums.ac.ir 
at this time frame and have a deeper impact on the patient and his family's life $[10,11]$.

\section{Materials and Methods}

Research population, place, and time: The study population included obese and overweight patients undergoing CABG surgery hospitalized in the cardiac surgery wards of the hospital affiliated to Guilan University of Medical Sciences in July to September 2018, in access manner $(\mathrm{N}=84)$.

A questionnaire containing four sections of individual and disease-related characteristics, Carlson Comorbidity Index (19 questions), Katz Index (16 questions in 7 sections) and Luton's Instrumental Activity of Daily Living (IADL) scale were used.

\section{Results}

Findings showed that most $\mathrm{p}$ patients were in $\mathrm{t}$ he following conditions: in the age group of 65 and under (79. 8\%), male (59. 5\%), married (98. 8\%), primary education (34. $5 \%$, left ventricular outflow fraction less than 50\% (82. $1 \%$ ), with more than two children (76. $2 \%$ ), with transplants more than 3 vessels (72. 6\%), no smoking (67.9\%)), and no carotid artery occlusion (70.2\%). According to the results, there was no significant relationship between Body Mass Index (BMI) and in the ADL and IADL status before and one month after surgery.

In the case of IADL, the variables entered the logistic regression model, in which the sex variables $(\mathrm{P}=0.004$, $\mathrm{OR}=10.51,95.1 \%=15.41)$, the carotid artery occlusion
$(\mathrm{P}=0.01,0.12=\mathrm{OR}, 0.64-0.62 \%=\mathrm{CI} 95 \%)$, and the IADL predictors showed obese and overweight in the patients. The men were 10.51 times more capable than women in practicing IADL a month later were. Patients with carotid artery occlusion were 0.12 times less likely than other patients.

\section{Discussion}

Obese or overweight people were able to get ADL one month after surgery. While for IADL they often needed help and dependency. This finding is consistent with the results of several studies in this case $[10,12,13]$. Because most male patients were around 59 years old, had an underlying disease, and because the economic responsibility and livelihood of the family are the responsibility of the men, these people are forced to return to work. A combination of these factors may cause financial and economic problems for the patient, healthcare systems, and society. In the case of more postoperative complications, overweight and obese patients are more likely to develop underlying conditions such as diabetes [14].

In the one month after surgery, there was a significant association between patients' ADL and their education, the length of cross-clamping of the thoracic aorta, the duration of use of the cardiopulmonary bypass pump, and the use of Beta blockers. The results of a study by Stamou et al. showed that the duration of use of cardiopulmonary bypass pump and cross-clamping pump of the aorta are influential variables [15].

The results of the study by Lehmkuhl et al. showed that the level of education is one of the influential factors in obese

Table 1. Estimation of regression coefficients of predictive factors related to Instrumental Activity Of Daily Living (IADL) during one month after surgery

\begin{tabular}{|c|c|c|c|c|c|c|}
\hline \multirow[t]{2}{*}{ Variables } & \multirow{2}{*}{$\begin{array}{l}\text { B Regression } \\
\text { Coefficient }\end{array}$} & \multirow{2}{*}{$\begin{array}{l}\text { S. E. Stan- } \\
\text { dard Error }\end{array}$} & \multirow[t]{2}{*}{ Sig. } & \multirow[t]{2}{*}{$\operatorname{Exp}(\beta)$} & \multicolumn{2}{|c|}{$\begin{array}{l}\text { 95\% Confidence Interval } \\
\text { for } \operatorname{Exp}(\beta)\end{array}$} \\
\hline & & & & & Lower & Upper \\
\hline Sex (male) & 2.35 & 0.81 & 0.004 & 10.51 & 2.15 & 51.41 \\
\hline Sex (female) & \multicolumn{6}{|c|}{ Reference } \\
\hline Left ventricular outflow fraction $(\leq 50)$ & 1.42 & 0.86 & 0.10 & 4.14 & 0.75 & 22.68 \\
\hline Left ventricular outflow fraction $(>50)$ & \multicolumn{6}{|c|}{ Reference } \\
\hline Carotid Artery Occlusion (Yes) & -2.07 & 0.83 & 0.01 & 0.12 & 0.02 & 0.64 \\
\hline Carotid Artery Occlusion (No) & \multicolumn{6}{|c|}{ Reference } \\
\hline Fixed value & 1.88 & 0.75 & 0.01 & 6.59 & - & - \\
\hline
\end{tabular}


people to perform ADL after surgery [16]. One month after the operation, there was a significant relationship between the status of ADL of patients with sex, occupation, education, smoking, and carotid artery occlusion. The results of a study by Najafi et al. indicated that sex is one of the influential factors in obese or overweight people $[17,18]$.

In connection with the predictive factors of the status of IADL within one month after Coronary Artery Bypass Grafting (CABG), the results showed that the sex and noncarotid artery occlusion in patients are predictive variables.

The results of a study showed that the sex of patients with obesity undergoing CABG surgery is one of the factors reducing the functional status of patients in the postoperative stage [19]. De Rosa et al. showed that people with overweight or obese who had carotid artery occlusion before surgery were less capable, and that $50 \%$ carotid artery occlusion was a predictive factor for mortality [20].

\section{Conclusion}

Obese and overweight people have a disability in their ADL performance, and it is important to pay attention to the predictors, including men and the carotid artery occlusion in their return to normal life after surgery.

\section{Restrictions and suggestions}

Using a questionnaire and telephone follow-up in one month after the operation and the low sample size are the limitations of this research. Instead of a questionnaire, the observation method with a different design and higher sample size can be used to examine the ability of IADL.

\section{Ethical Considerations}

\section{Compliance with ethical guidelines}

This study was approved (Ethics Code: IR.GUMS. REC.1395.86) in the ethics committee of Guilan University of Medical Sciences.

\section{Funding}

The present paper was extracted from the MSc. thesis of the first author, Department of Nursing, School of Nursing and Midwifery, Guilan University of Medical Sciences and supported financially by the Vice Chancellor for Research and Technology of Guilan University of Medical Sciences (Code: 27272).

\section{Authors' contributions}

All authors contributed in preparing this article.

\section{Conflicts of interest}

The authors declared no conflict of interest.

\section{Acknowledgements}

The authors would like to thank all the officials of Guilan University of Medical Sciences for permiting to conduct the research, as well as the patients participated in the cardiac surgery department in this study. 


\title{
بررسى عوامل بيشبينى إننده فعاليتهاى روزمره و ابزارى زندتى در بيماران هاق و داراى

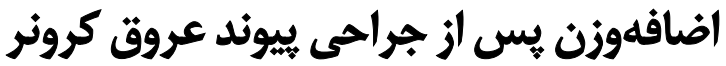

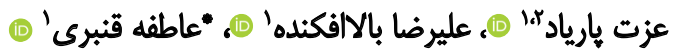

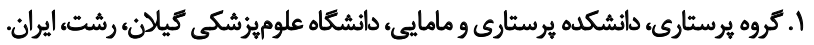

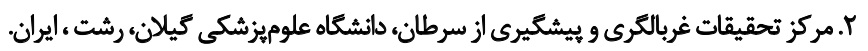

\begin{abstract}
حكيد

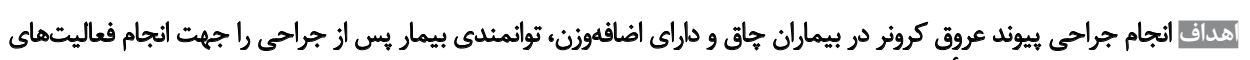

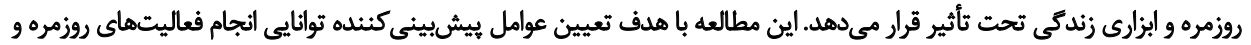
ابزارى زندكى انجام شده است.

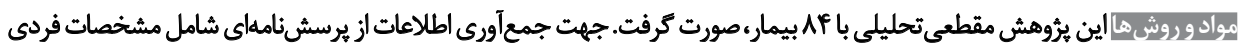

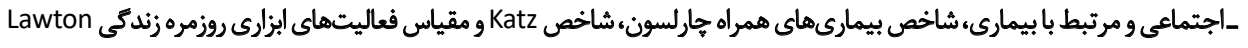

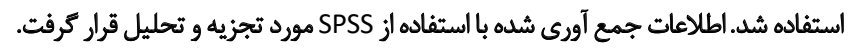

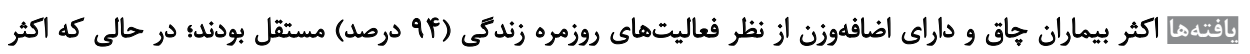

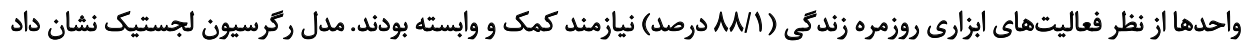

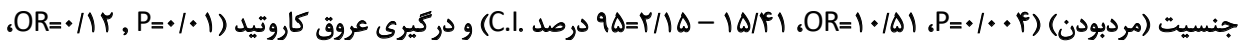

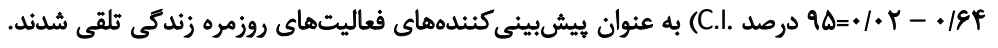

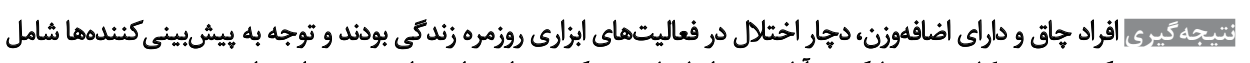

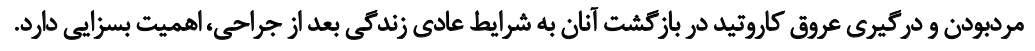

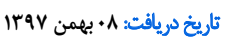

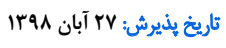

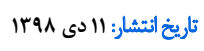

كليدوأوها:

فعاليتهاى روزمره

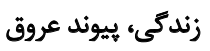
كرونر، شاخص توند تونه بدنى، فعاليتهاى ابزارى توني روزمره زندكى فيتى

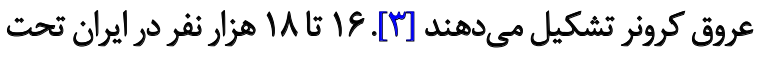

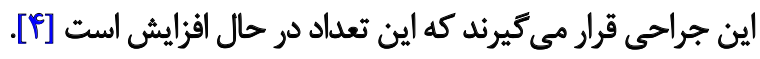

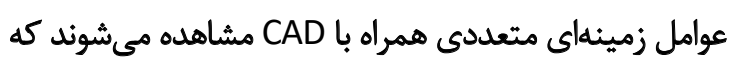

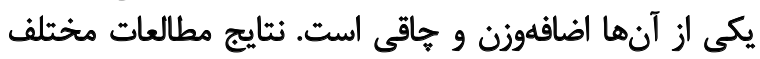

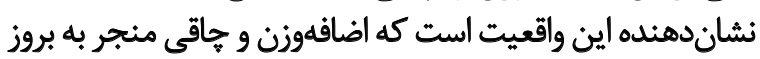

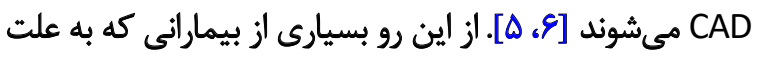

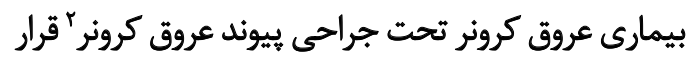

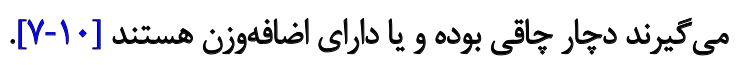

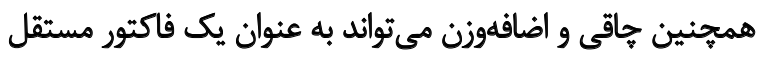

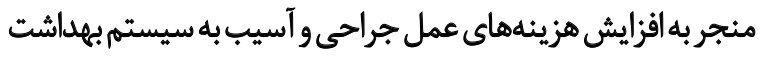

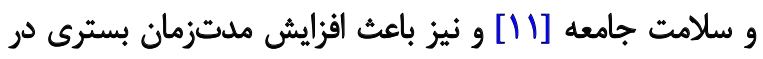

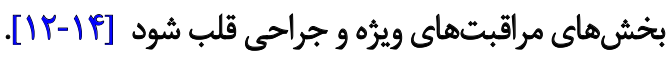

2. Coronary Artery Bypass Graph (CABG)

مقدمه

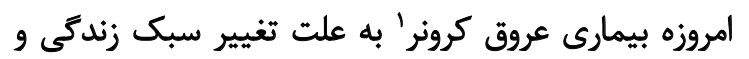

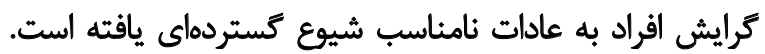

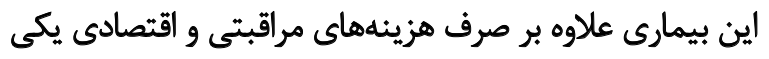

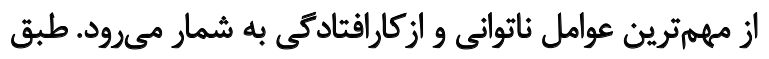

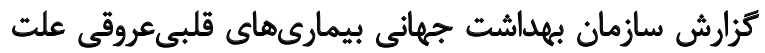

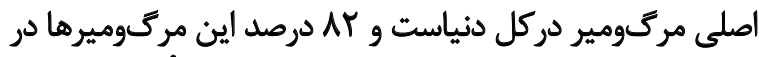

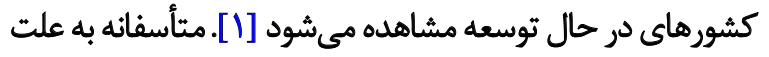

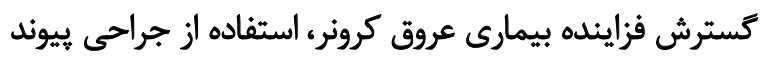

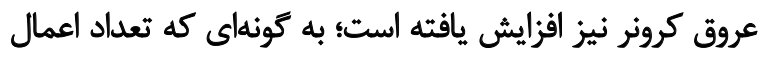

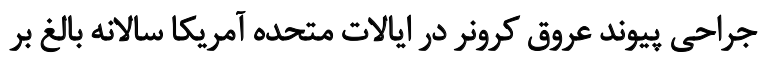
از أ TITV. .

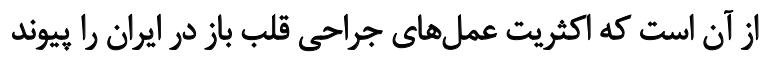

$$
\text { ……... }
$$

1. Coronary Artery Disease (CAD)

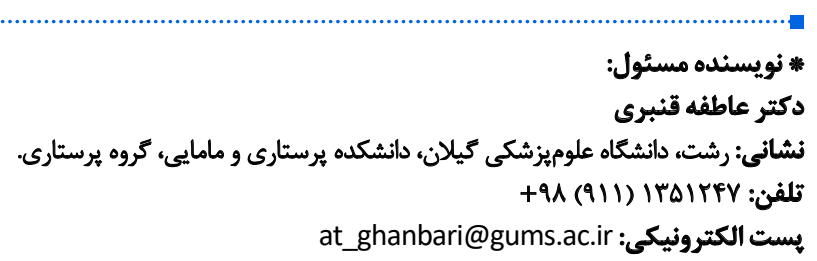




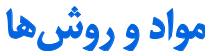

اين يُروهش يك يُروهش مقطعى از نوع تحليلى است. جامعه اين

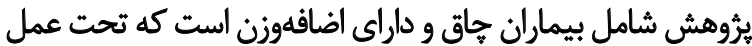

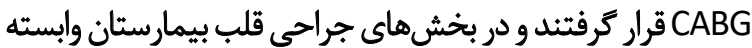

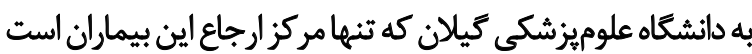

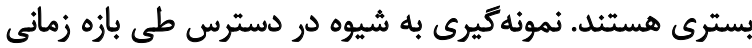

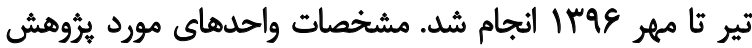

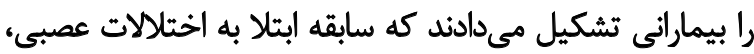

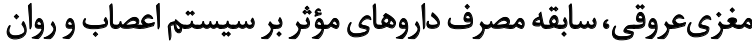

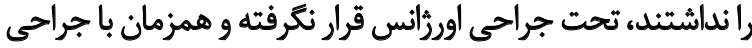
بيوند عروق كرونر، جراحى عروق كاروتيد نيز نداشتند. نبود هركونه مشكل جسمى ـ ظاهرى (اختلالات حركتى،

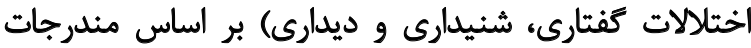

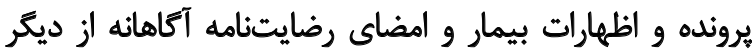

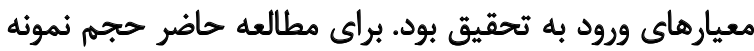

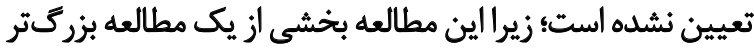

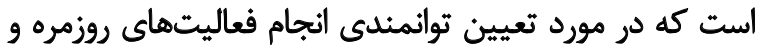

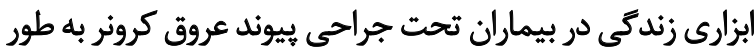

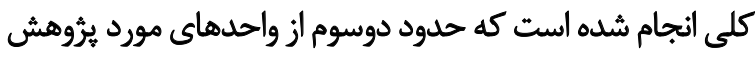

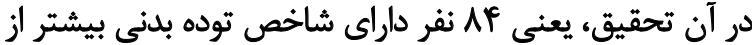

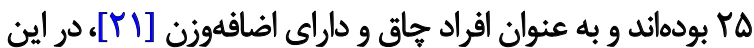
مطالعه مورد بررسى قرار ترفيتند.

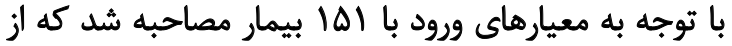

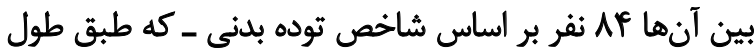

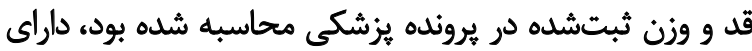

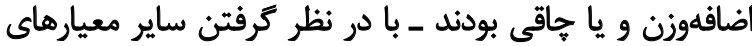

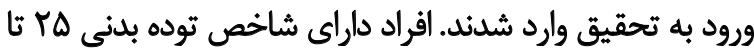

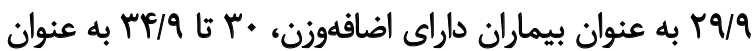

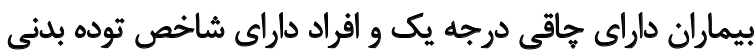

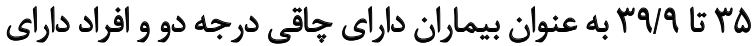

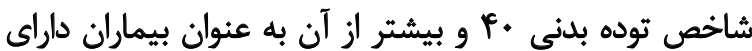

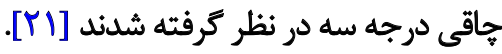

جهت جمعآورى اطلاعات در اين ثروهش از يرسشنامداى

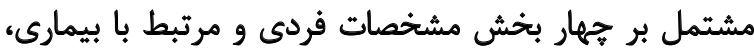

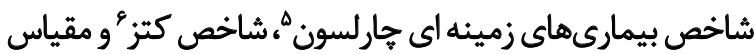

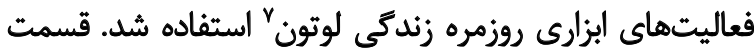

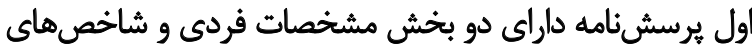

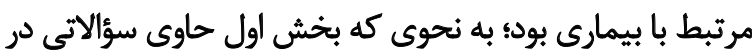

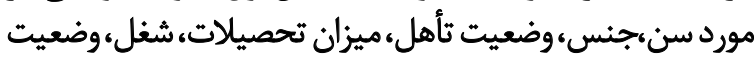

\section{Charlson Comorbidity Index}

6. Katz Index

7. The Lawton Instrumental Activities of Daily Living Scale
مئتواند استقلال عمل اين افراد راكه بسيارى از آنها

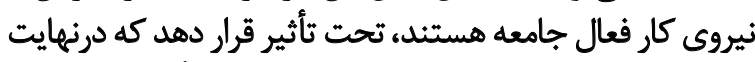

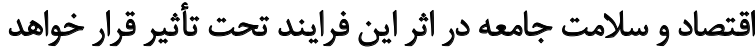

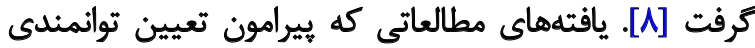

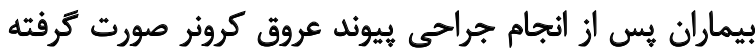

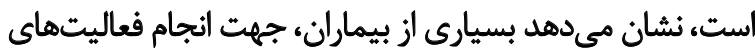

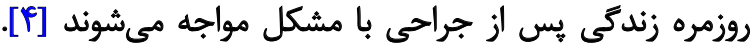

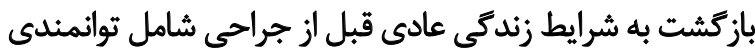
انجام فعاليتهاى ابزارى و روزمره زندكى عاسي است.

منظور از فعاليتهاى روزمره زندكَى" توانايى بيمار در انجام

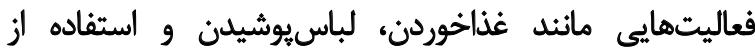

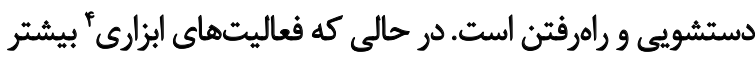

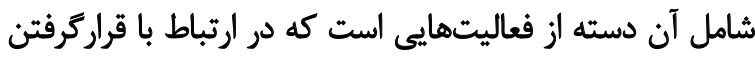

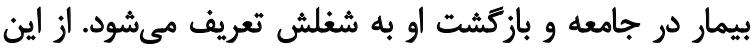

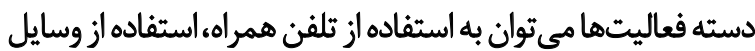

حمل ونقل و توانايى جهث انجام محاسبات مالى اشاره كرد [1ه].

با توجه به مطالعات مختلف عوامل متفاوتى مى توانند بر وضعيت

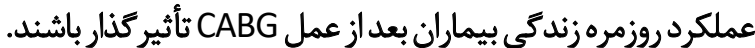

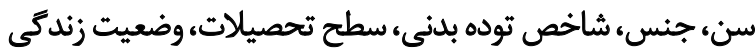

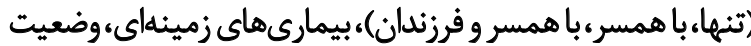

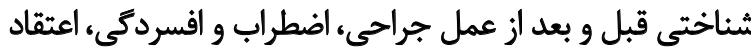

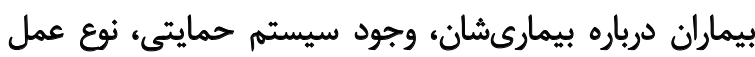

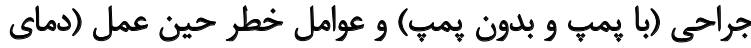

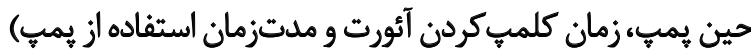

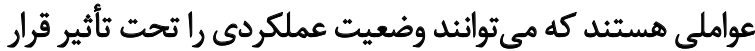

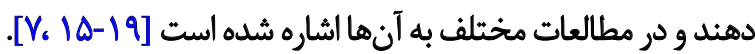
بديهى است كه توانمندى بيماران جهت انجام مراقبتهاى

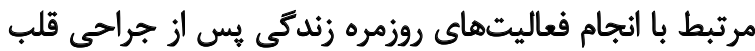

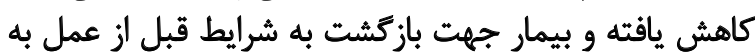

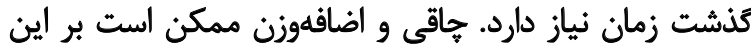

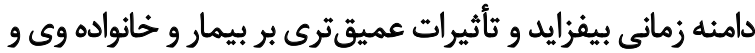

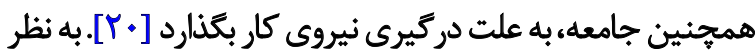

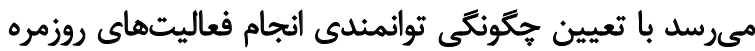

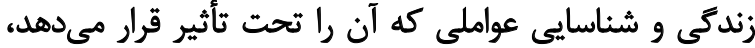

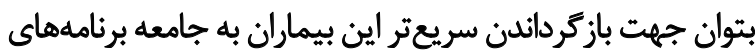

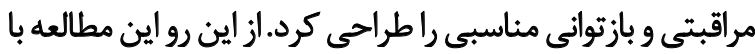

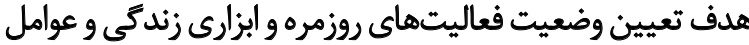

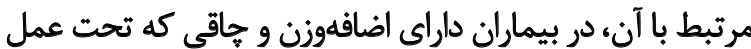
جراحى ييوند عروق كرونر قرار ترفتهانده انجام شده است إن.

3. Activity Daily Living (ADL)

4. Instrumental Activity Daily Living (IADL) 
متغير وابسته تحقيق يعنى وضعيت فعاليتهاى روزمره و ابزارى

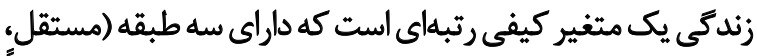

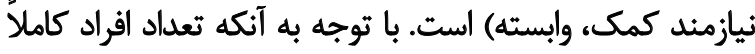

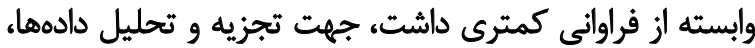

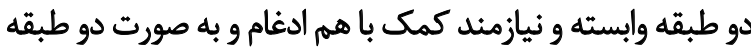

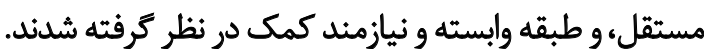

Ldî́?

يافتههاى اين تحقيق نشان داد كه اكثر واحدهاى مورد

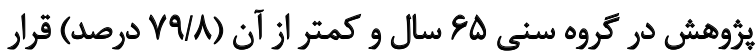

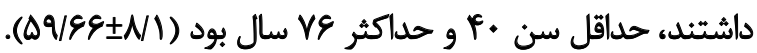

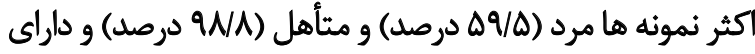

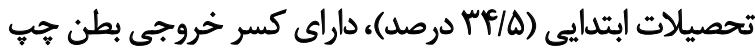

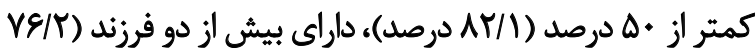

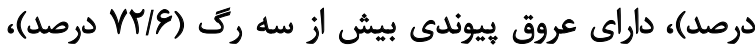

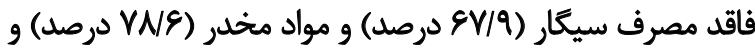

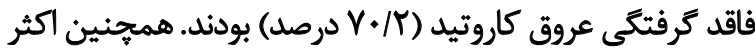

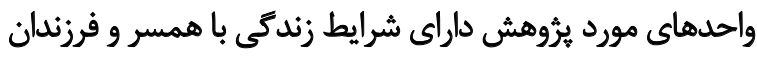
(TF)

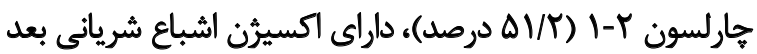

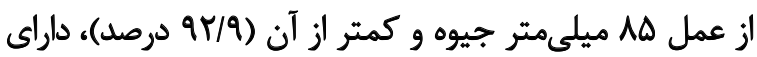

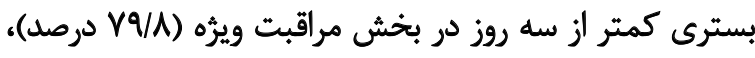

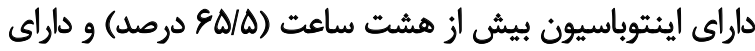

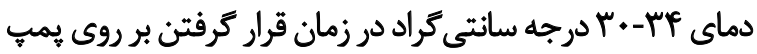

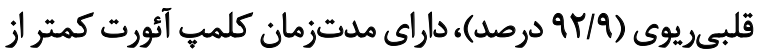

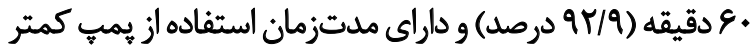

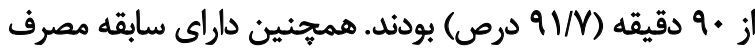

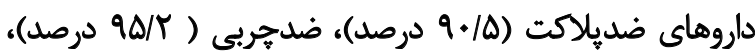

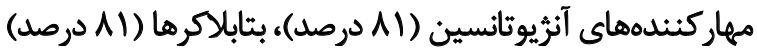
و نيتراتها (99 درصد) بوندين.

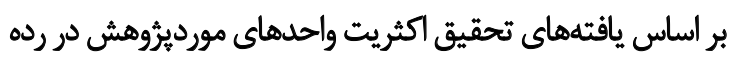

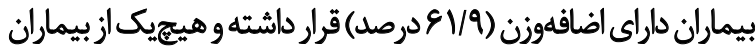

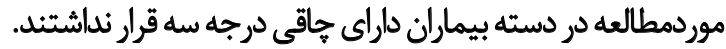
با توجه به آنكه در مرحله قبل از عمل هيجيك از بيماران

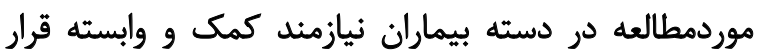

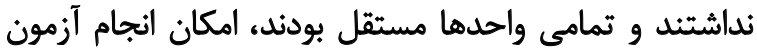

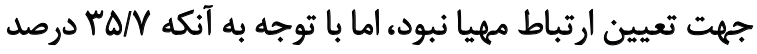

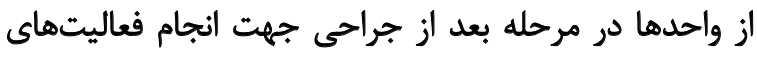

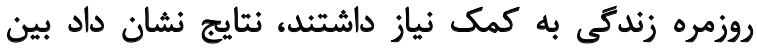

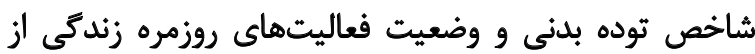

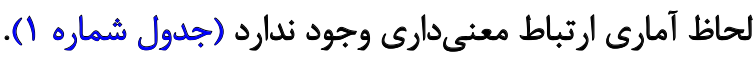

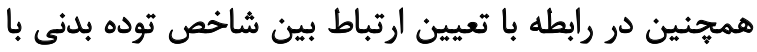

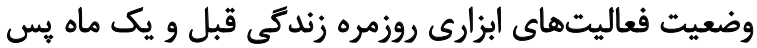

زندكَى، شاخص توده بدنى و بخش دوم حاوى سؤالاتى در مورد

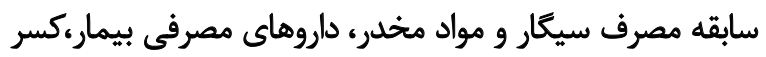

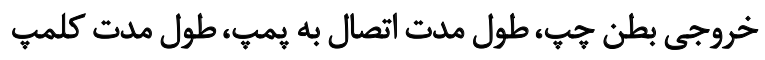

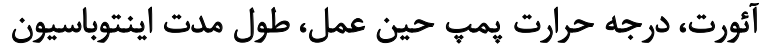

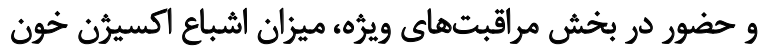

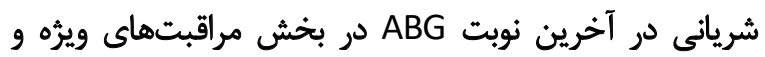
كرفتخى عروق كاروتيد بود. قسمت دوم يرسشنامه شامل شاخص بيمارىهاى همراه

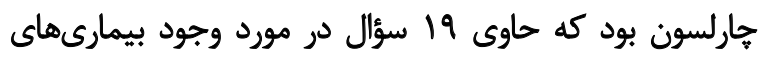

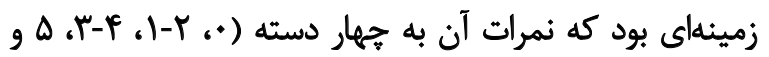

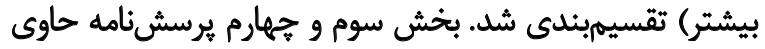

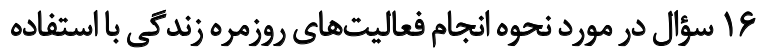

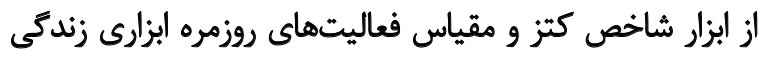

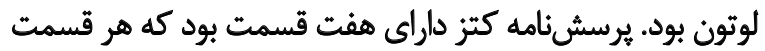

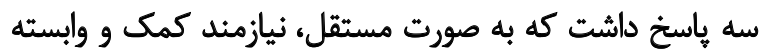

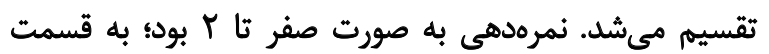

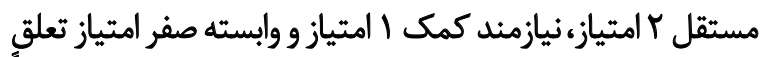

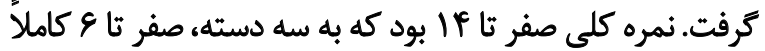

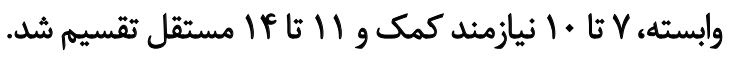
توانايى انجام فعاليتهاى روزمره ابزارى از طريق مقياس لوتون -

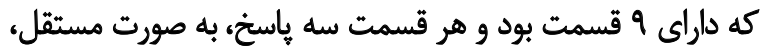

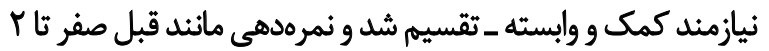

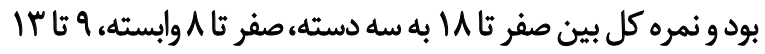

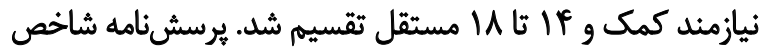

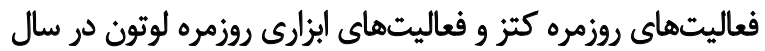

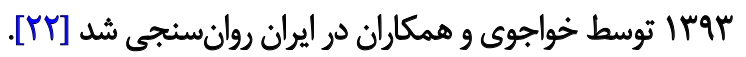
ابزارهاى كردآورى اطلاعات در دو مرحله (قبل و يك ماه هوريس

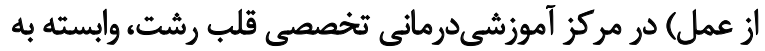

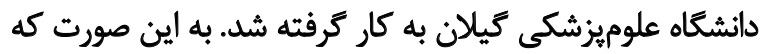

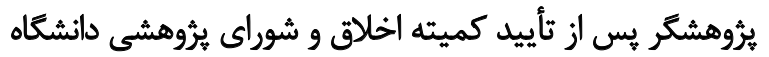

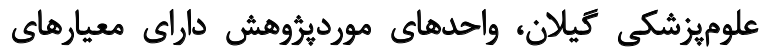

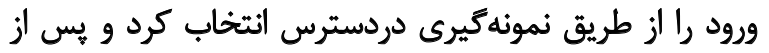

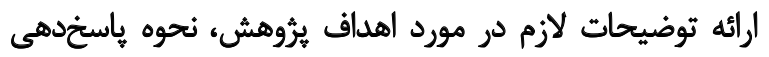

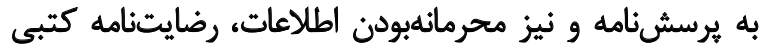

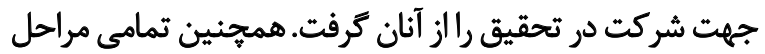

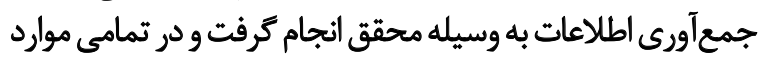

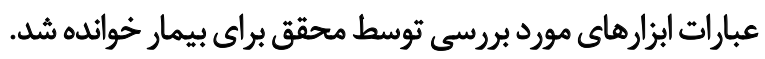

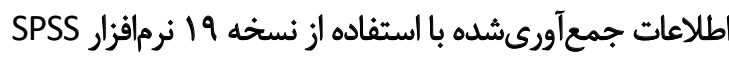

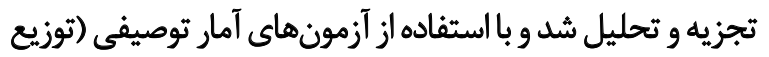

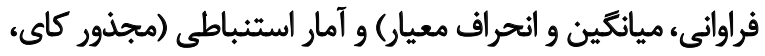

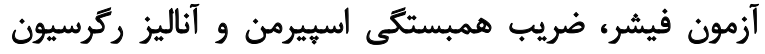

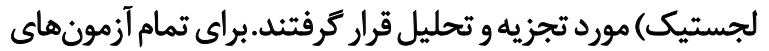

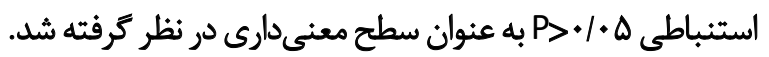


جدول ا. ارتباط بين وضعيت فعاليتهاى روزمره زندكى با شاخص توده بدنى

\begin{tabular}{|c|c|c|c|}
\hline \multirow{2}{*}{ مستقل } & يك ماه هيس از عمل & قبل از عمل & \multirow{2}{*}{ وضعيت فعاليتهاي } \\
\hline & وابسته و نيازمثد كمك & مستقل & \\
\hline \multicolumn{3}{|c|}{ تعداد (درصد) } & \\
\hline$p q(\Delta N \mathbb{N})$ & $r(r / s)$ & $\operatorname{ar}(\varepsilon \vee / q)$ & اضافهوزن \\
\hline$r \cdot(r \Delta / V)$ & $r(T / P)$ & $M(Y N)$ & جاقي \\
\hline
\end{tabular}

$P=* / /$ re*

$\mathrm{P}=\cdot / \mathrm{r} \mathrm{\Lambda}^{*}$

توع آزمون و قضاوت

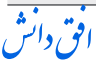

Spearman's test*

Chi-square test**

جدول r. ارتباط بين وضعيت فعاليتهاى ابزارى روزمره زندكى با شاخص ثوده بدنى

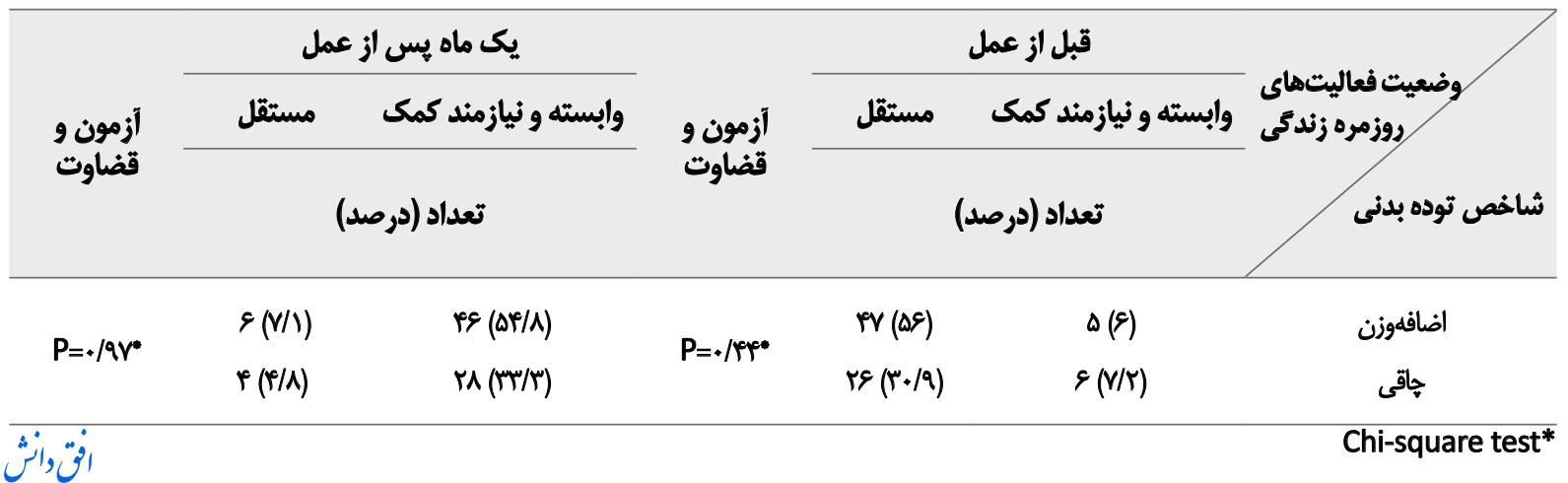

مجذور كاى و فيشر هيجزونه ارتباط معنى دارى رابين متغيرهاى از عمل، نتايج نشان داد كه بين شاخص توده بدنى و وضعيت

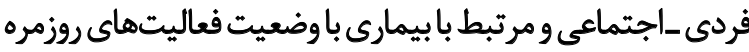

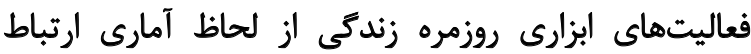

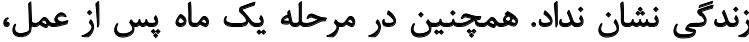

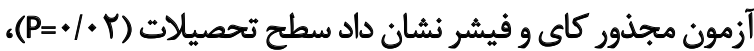

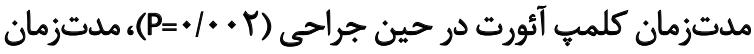

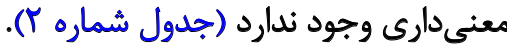

در ارتباط بين تعيين عوامل فردى ـ اجتماعى و مرتبط با فيا

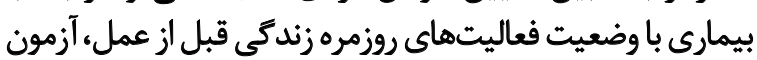

جدول ب. توزيع وضعيت فعاليتهاى روزمره زندكى يك ماه بعد از عمل بر حسب متغيرهاى فردى ـاجتماعى و مداخله

\begin{tabular}{|c|c|c|c|c|}
\hline$P$ & مستقل & وابسته و نيازمثد كمك & 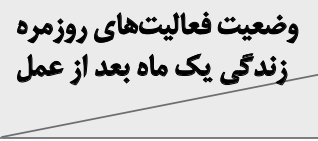 & مثغيرها \\
\hline \multirow{2}{*}{$+1+r^{\circ}$} & & $f(f / \lambda)$ & زير دييلم & \multirow{2}{*}{ ميزان تحصيلات } \\
\hline & $M(M f / T)$ & $1(N / T)$ & دييلم و بالاتر & \\
\hline \multirow{2}{*}{ Hen } & $V \&(9 \cdot 10)$ & $r(r / F)$ & g.> & \multirow{2}{*}{ طول مدت كراس كلمب آثورت } \\
\hline & $r(r / \varphi)$ & $r(r / q)$ & $8 \cdot 2$ & \\
\hline \multirow{2}{*}{$.1 . . \mu * *$} & $V \Delta\left(A q /{ }^{\prime}\right)$ & $r(r / F)$ & $9+>$ & \multirow{2}{*}{ طول هلت اسثقاده از بميمي حين } \\
\hline & $f(F / A)$ & $r(r / 9)$ & $9+2$ & \\
\hline \multirow{2}{*}{$+/ *+\infty$} & E\& (VNF) & $r(T / P)$ & 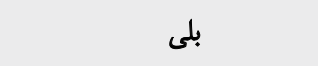 & \multirow{2}{*}{ مصرف داروهاى بثا بلاكر } \\
\hline & $\|(1 Q / \Delta)$ & $r(r / s)$ & خير & \\
\hline
\end{tabular}

Fisher's Exact Test** 
جدول F. برآورد ضرايب ركرسيونى عوامل بيشبينى كنينده مرتبط با فعاليتهاى ابزارى روزمره زندكى يك ماه يس از عمل

\begin{tabular}{|c|c|c|c|c|c|c|}
\hline \multicolumn{2}{|c|}{ 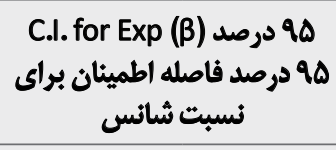 } & \multirow[t]{2}{*}{ 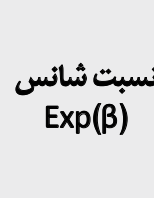 } & \multirow[t]{2}{*}{$\begin{array}{l}\text { معنى دارى } \\
\text { Sig. }\end{array}$} & \multirow[t]{2}{*}{ استانداردى خطاى } & \multirow[t]{2}{*}{ ركريبون B ريب } & \multirow[t]{2}{*}{ مثغير ها } \\
\hline حد بالا & حد بايين & & & & & \\
\hline$\Delta V / F i$ & $r / N Q$ & $1 . / 01$ & $\%+. .4$ & $\cdot|\Lambda|$ & T/TQ & جنس (مرد) \\
\hline \multicolumn{5}{|c|}{ رفرنس } & \multicolumn{2}{|r|}{ جنس (مؤنث) } \\
\hline MT/FA & .NA & $F / N f$ & 八. & $\cdot \mathbb{N}$ & D/er & كسر خروجى بطن جي (ه.z) \\
\hline \multicolumn{6}{|c|}{ رفرنس } & كسر خروجى بطن جِي (>-A) \\
\hline .199 & $.1 . r$ &.$/ 1 r$ & .1 .1 & / Ar & $-Y / \cdot V$ & دركيرى عروق كاروتيد (بلم) \\
\hline \multicolumn{6}{|c|}{ رفرنس } & دركيرى عروق كاروتيد (خير) \\
\hline- & - & $8 / 109$ & $.1 \% 1$ &.$/ N \Delta$ & $V / M$ & مقدار ثابت \\
\hline
\end{tabular}

\section{بحث و نتيجهئيرى}

يُورهش حاضر با هدف تعيين وضعيت فعاليتهاي روزمره و

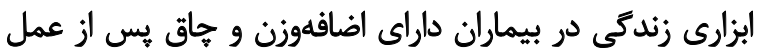

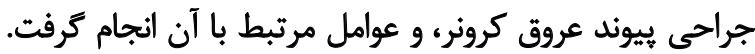

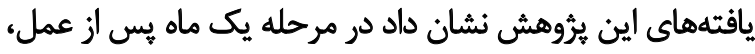

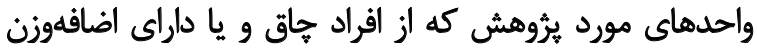

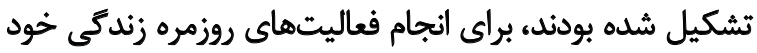

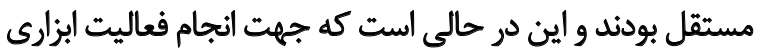

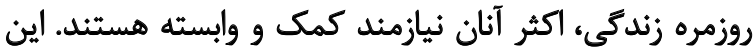

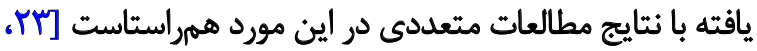

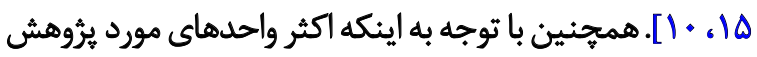

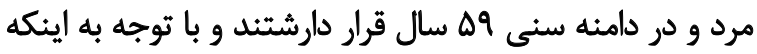

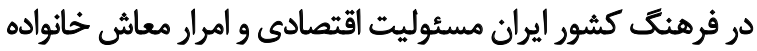

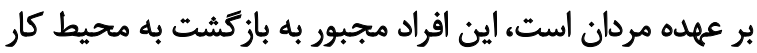

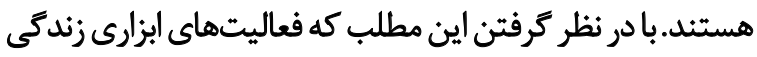

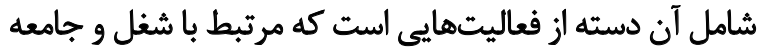

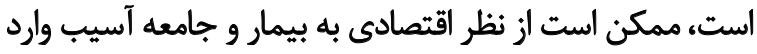

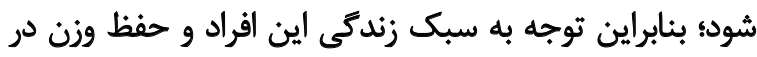
دامنه طبيعى، ضرورى به نظر مىرسد. توريك

همجنين يافتههاى اين يروهش نشان داد اكثر واحدهاي مورد

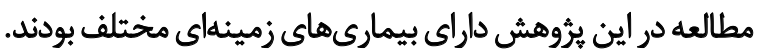

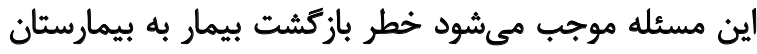

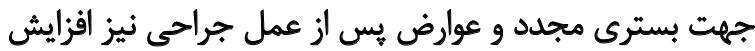

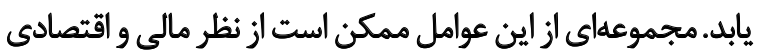

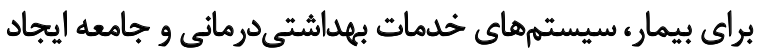
معضل كند. در راستاى يافتههاى اين تحقيق در مورد عوارض جيتي

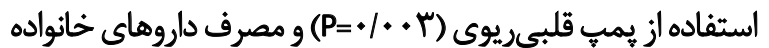

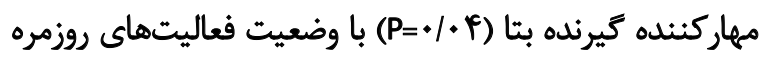

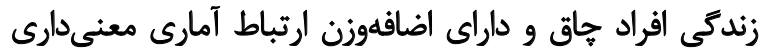

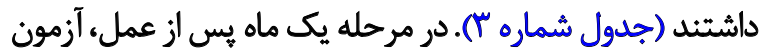

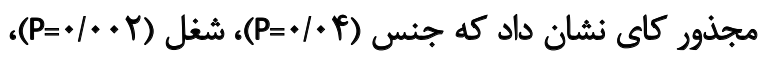

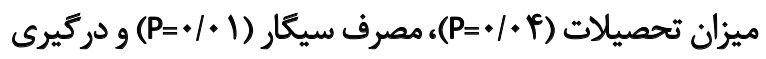

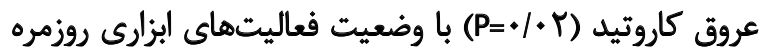
زندكى ارتباط آمارى داشتند.

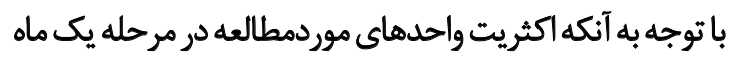

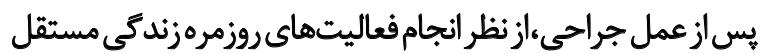

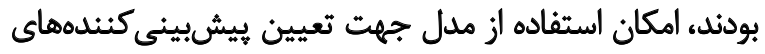

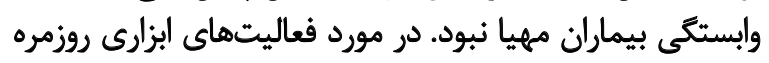

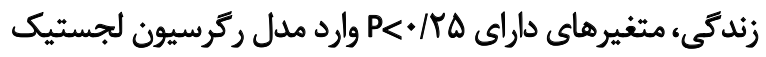

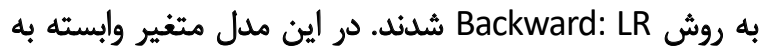

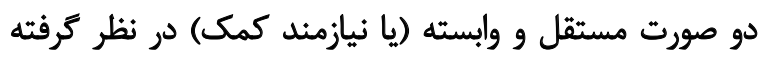

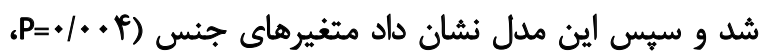

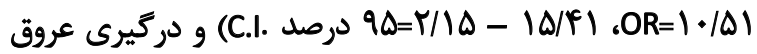

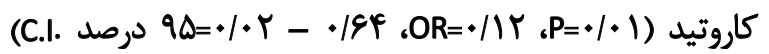

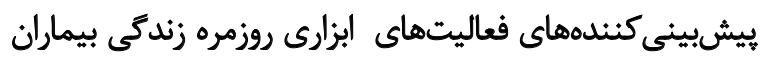

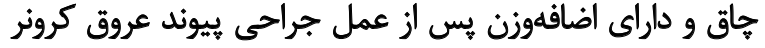

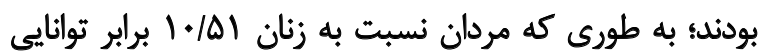

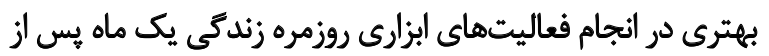

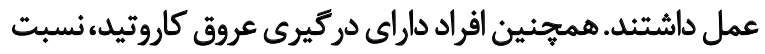

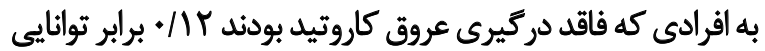

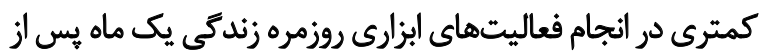

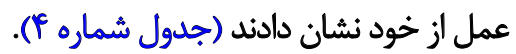


يافتههاي ديكر اين تحقيق مؤيد آن است كه در مرحله يك ماه

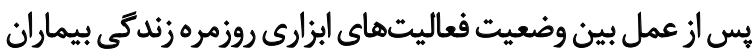

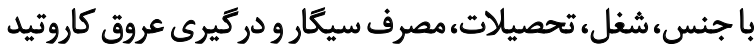

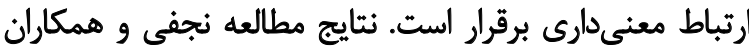

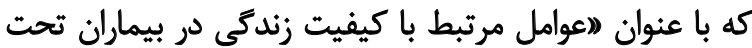

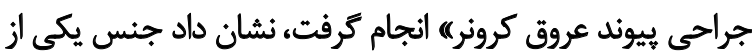

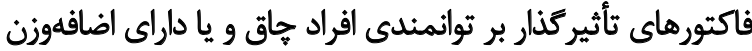

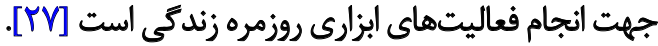

مطالعه حسينى و همكاران با هدف تعيين ارتباط بيمارى

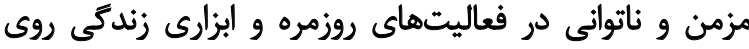

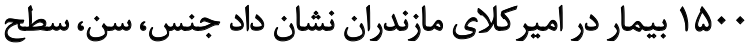

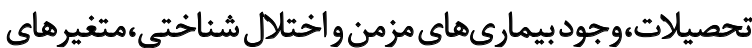

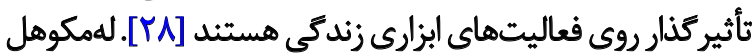

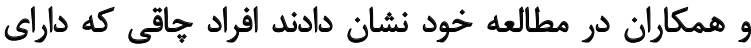

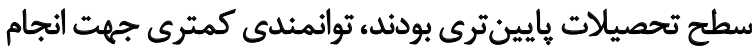

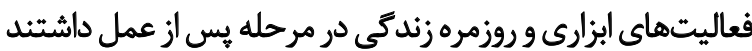

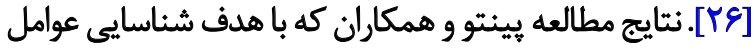

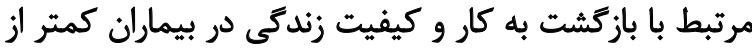

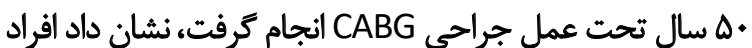

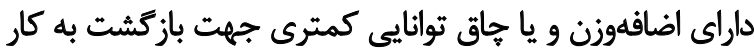

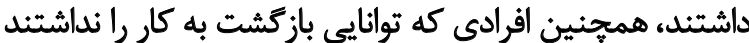

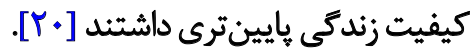

نتايج مطالعه وانك و همكاران كه با هدف شناسايى عوامل

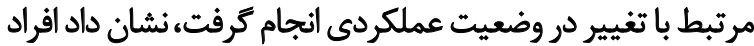

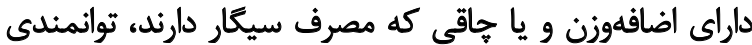

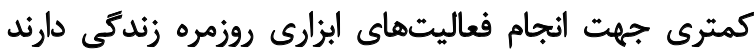

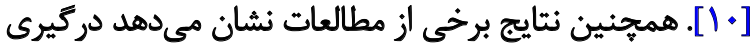

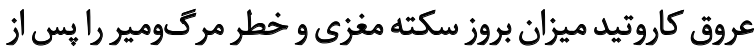

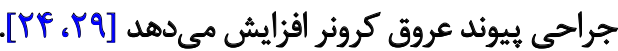

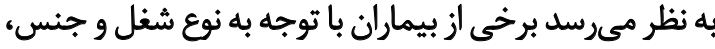

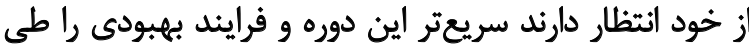

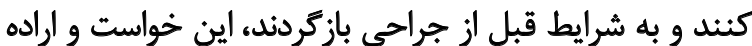

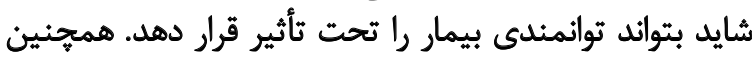

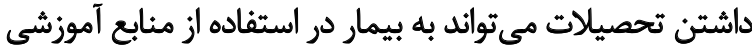
مختلف در جهت بهبودى سريعتر كمك كند.

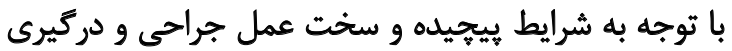

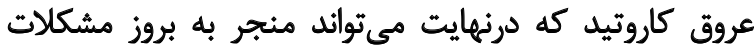

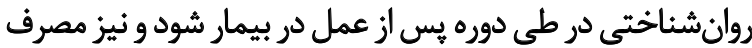

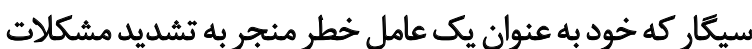

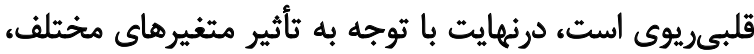

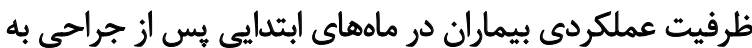

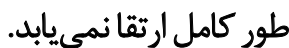

بيشتر يس از جراحي، معمولاً بيماران داراي اضافهوزن و جاقيت

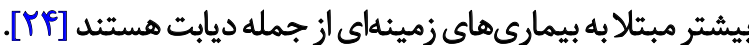

يافتههاى اين تحقيق نشان داد در مرحله يك ماه يس از براز

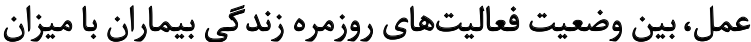

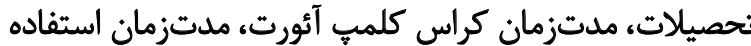

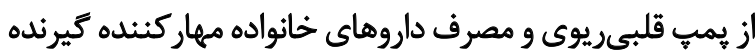

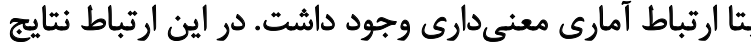

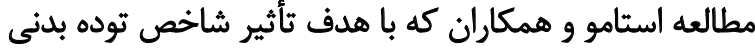

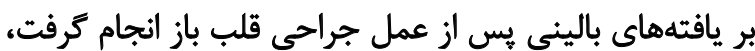

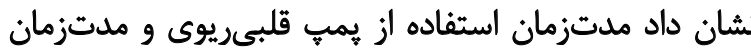

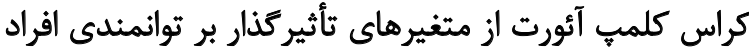

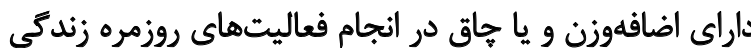

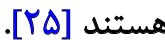

همجِين نتايج مطالعه لهمكوهل و همكاران كه با هدف

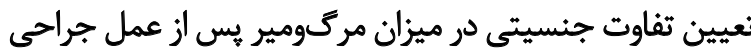
انجام شد، نشان داد سطح تحصيلات از عوامل تأثير مئدار

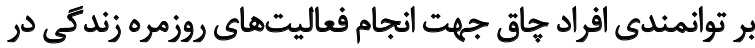

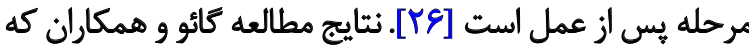

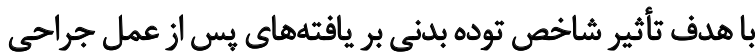

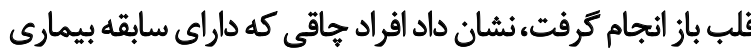

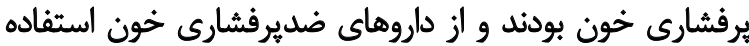

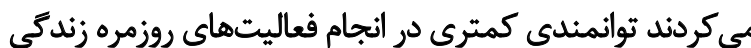

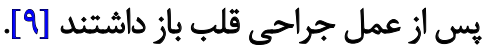
به نظر مىرسد طولانىبودن مدتزمان كراس كلمب آئورت

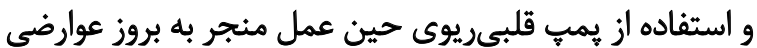

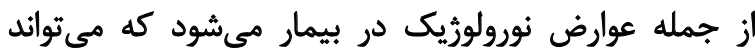

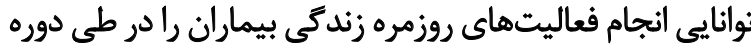

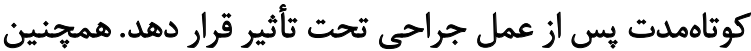

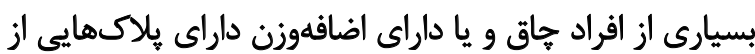

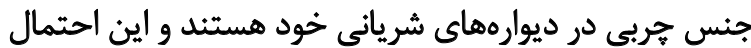

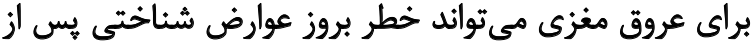

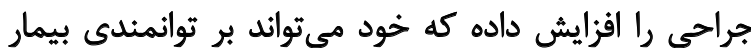

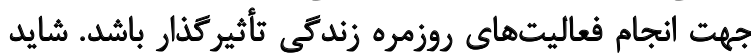

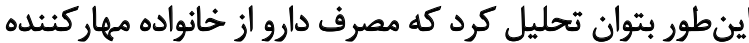

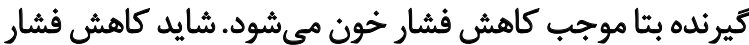

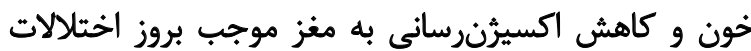

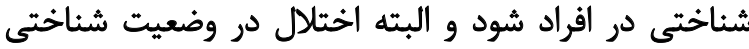

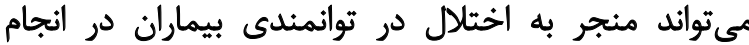

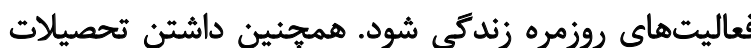

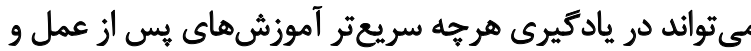

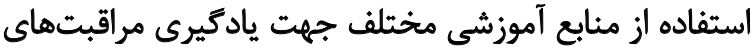

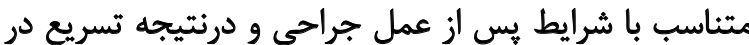
فرايند بجبودى كمك كنينده باشد. 


\section{ملاحظات اخلاقى

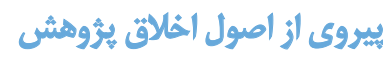

اين مطالعه با كداخلاق IR.GUMS.REC.1395.86 اخر كميته اخلاق دانشكاه علوميزشكى كيلان مورد ماند تأييد قرار كرفت.

\section{لمافي}

اين بروهش باحمايت مالى معاونت تحقيقات و فناورى دانشعاه

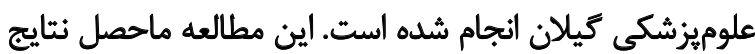

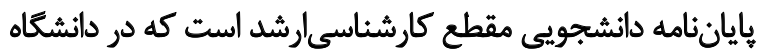

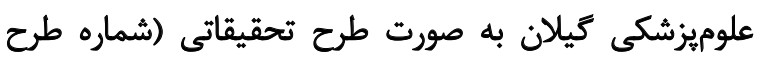

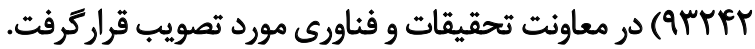

\section{مشاركت نويسئد مكان}

تمامى نويسندكان در آمادهسازى اين مقاله مشاركت داشتهاند.

$$
\text { تقارض منأع }
$$

نويسندُّان اظهار مي دارند هيجّونه تعارض منافعى در اين

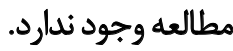

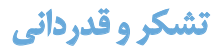

يُروهشكران از تمامى مسئولين دانشكاه علوميزشكى كيلان به

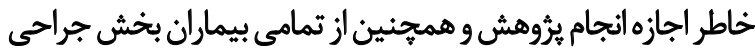

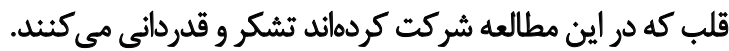

در ارتباط باعوامل بيشبينى كننده وضعيت فعاليتهاي ابزارى

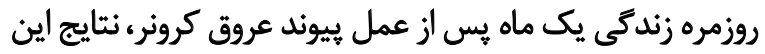

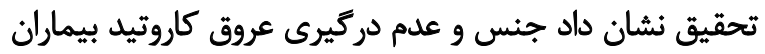

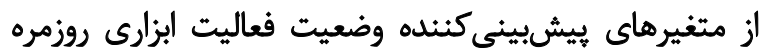

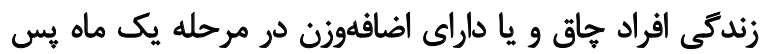

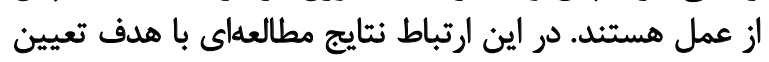

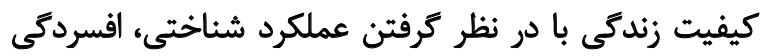

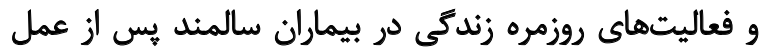

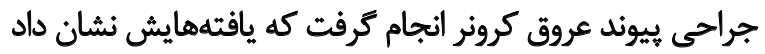

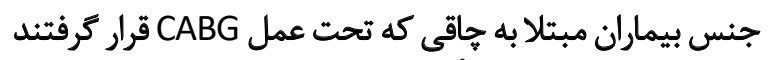

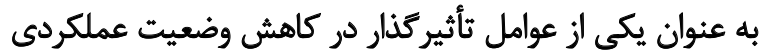

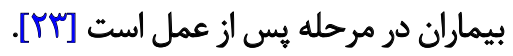

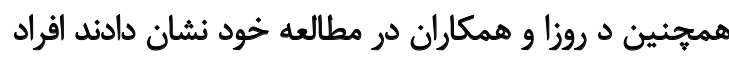

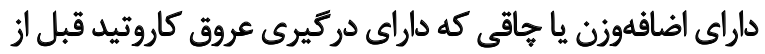

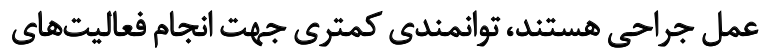

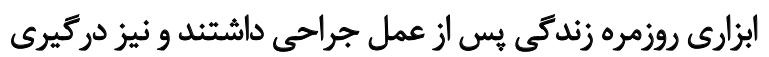

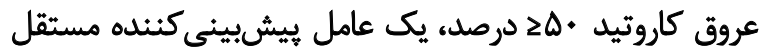

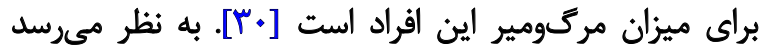

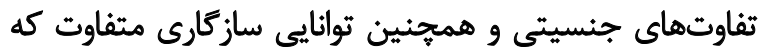

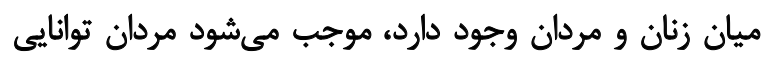

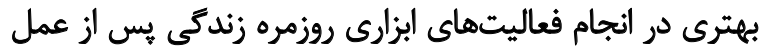

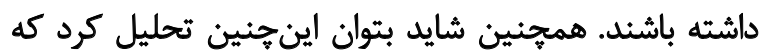

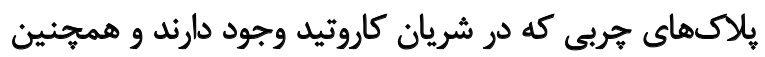

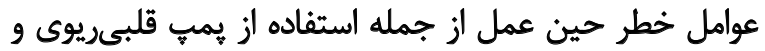

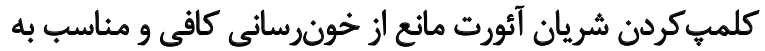

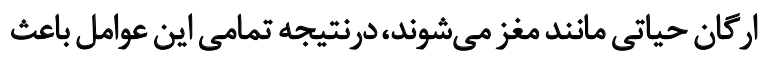

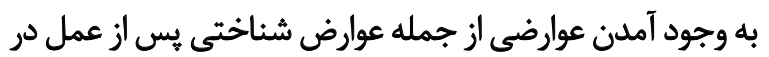

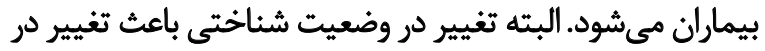

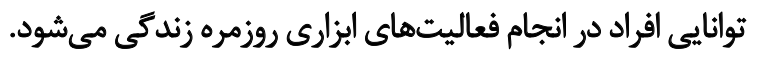

يكى از محدوديتهاي اين تحقيق استفاده از يرسش إنامه براي

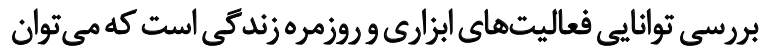

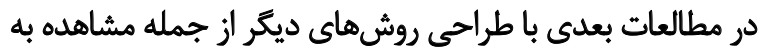

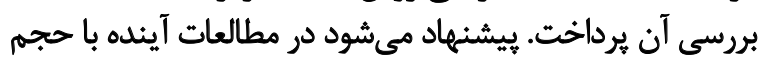

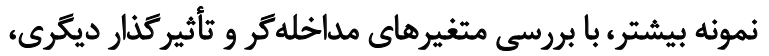

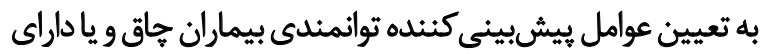

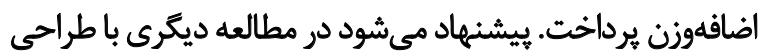

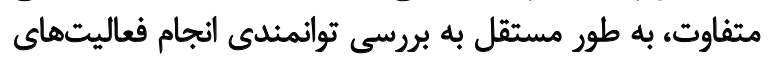

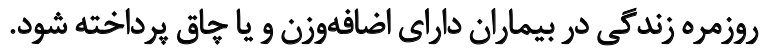

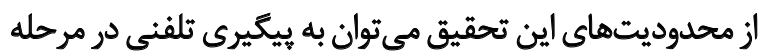

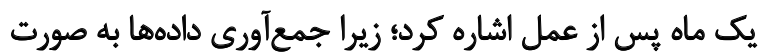
تلفنى ممكن است منعكس كننده شرايط واقعى بيمار نبر نباشد. 


\section{References}

[1] Siavoshi S, Roshandel M, Zareiyan A, Ettefagh L. [The effect of cardiac rehabilitation care plan on the quality of life in patients undergoing coronary artery bypass graft surgery (Persian)]. Cardiovascular Nursing Journal. 2012; 1(2):38-46. http://journal.icns.org.ir/article-1-74-en.html

[2] Weiss AJ, Elixhauser A. Trends in operating room procedures in U.S. hospitals, 2001-2011: Statistical brief \#171. Rockville, MD: Agency for Healthcare Research and Quality (US); 2006. [PMID]

[3] Paryad E, Rouhi Balasi L. Smoking cessation: Adherence based on patients' illness perception after coronary artery bypass grafting surgery. Indian Heart J. 2018; 70(Suppl. 3):S4-7. [DOI:10.1016/j.ihj.2018.01.025]

[4] Raiesdana N, Kamali E, Soleimani M, Mir Mohammadkhani M. [Assessment of situational and heart focused anxiety in patients with coronary artery disease before angiography (Persian)]. Koomesh. 2017; 19(1):199206. http://koomeshjournal.semums.ac.ir/article-1-3260-en.html

[5] Benjamin EJ, Blaha MJ, Chiuve SE, Cushman M, Das SR, Deo R, et al. Heart disease and stroke statistics-2017 update: A report from the American Heart Association. Circulation. 2017; 135(10):e146-e603. [DOI:10.1161/CIR.0000000000000485]

[6] Beukers NG, van der Heijden GJ, van Wijk AJ, Loos BG. Periodontitis is an independent risk indicator for atherosclerotic cardiovascular diseases among 60174 participants in a large dental school in the Netherlands. Journal of Epidemiology and Community Health. 2017; 71(1):37-42. [DOI:10.1136/jech-2015-206745] [PMID] [PMCID]

[7] Benedetto U, Caputo M, Zakkar M, Davies A, Gibbison B, Bryan A, et al. The effect of obesity on survival in patients undergoing coronary artery bypass graft surgery who receive a radial artery. European Journal of Cardio-Thoracic Surgery. 2016; 51(2):376-81. [DOI:10.1093/ejcts/ ezw323] [PMID] [PMCID]

[8] Costa VE, Ferolla SM, Reis TO, Rabello RR, Rocha EA, Couto CM, et al. Impact of body mass index on outcome in patients undergoing coronary artery bypass grafting and/or valve replacement surgery. Brazilian Journal of Cardiovascular Surgery. 2015; 30(3):335-42. [DOI:10.5935/16789741.20150027] [PMID] [PMCID]

[9] Gao M, Sun J, Young N, Boyd D, Atkins Z, Li Z, et al. Impact of body mass index on outcomes in cardiac surgery. 8. 2017; 61(1):2-3. [DOI:10.1097/01.sa.0000513504.81349.91]

[10] Wang M, Chen M, Ao H, Chen S, Wang Z. The effects of different BM on blood loss and transfusions in Chinese patients undergoing coronary artery bypass grafting. Annals of Thoracic and Cardiovascular Surgery. 2017; 23(2):83-90. [DOI:10.5761/atcs.0a.16-00219] [PMID] [PMCID]

[11] Johnson AP, Parlow JL, Milne B, Whitehead M, Xu J, Rohland S, et al. Economies of scale: Body mass index and costs of cardiac surgery in Ontario, Canada. The European Journal of Health Economics. 2017; 18(4):471-9. [DOI:10.1007/s10198-016-0803-4] [PMID]

[12] Almashrafi A, Alsabti H, Mukaddirov M, Balan B, Aylin P. Factors associated with prolonged length of stay following cardiac surgery in a major referral hospital in Oman: A retrospective observational study. BMJ Open. 2016; 6(6):e010764. [DOI:10.1136/bmjopen-2015-010764] [PMID] [PMCID]

[13] Terada T, Johnson JA, Norris C, Padwal R, Qiu W, Sharma AM, et al. Severe obesity is associated with increased risk of early complications and extended length of stay following coronary artery bypass grafting surgery. Journal of the American Heart Association. 2016; 5(6):e003282. [DOI:10.1161/JAHA.116.003282] [PMID] [PMCID]

[14] Masoumi SZ, Kazemi F, Khani S, Seifpanahi-Shabani H, Garousian M, Ghabeshi M, et al. Evaluating the effect of cardiac rehabilitation care plan on quality of life of patients undergoing coronary artery bypass graft surgery. International Journal of Cardiovascular Practice. 2017; 2(2):44-50. [DOI:10.21859/ijcp-020204]

[15] Jekel K, Damian M, Wattmo C, Hausner L, Bullock R, Connelly PJ, et al. Mild cognitive impairment and deficits in instrumental activities of daily living: A systematic review. Alzheimer's Research \& Therapy. 2015; 7(1):17. [DOI:10.1186/s13195-015-0099-0] [PMID] [PMCID]

[16] Hogue CW Jr, Fucetola R, Hershey T, Nassief A, Birge S, Dávila-Román VG, et al. The role of postoperative neurocognitive dysfunction on quality of life for postmenopausal women 6 months after cardiac surgery. Anesthesia and Analgesia. 2008; 107(1):21-8. [DOI:10.1213/ ane.0b013e3181606a65] [PMID] [PMCID]

[17] Rantanen A, Tarkka MT, Kaunonen M, Tarkka M, Sintonen H, Koivisto $A M$, et al. Health-related quality of life after coronary artery bypass grafting. Journal of Advanced Nursing. 2009; 65(9):1926-36. [DOI:10.1111/ j.1365-2648.2009.05056.x] [PMID]

[18] Seyam Sh, Heidarnia AR, Sadat Tavafian S. [Quality of life and factors related to it in cardiovascular patients after heart surgery (Persian)]. Journal of Birjand University of Medical Sciences. 2013; 19(6):33-41. http://journal.bums.ac.ir/article-1-1227-fa.html

[19] Juergens MC, Seekatz B, Moosdorf RG, Petrie KJ, Rief W. Illness beliefs before cardiac surgery predict disability, quality of life, and depression 3 months later. Journal of Psychosomatic Research. 2010; 68(6):553-60. [DOI:10.1016/j.jpsychores.2009.10.004] [PMID]

[20] Pinto N, Shah P, Haluska B, Griffin R, Holliday J, Mundy J. Return to work after coronary artery bypass in patients aged under 50 years. Asian Cardiovascular and Thoracic Annals. 2012; 20(4):387-91. [DOI:10.1177/0218492312437881] [PMID]

[21] Romero-Corral A, Montori VM, Somers VK, Korinek J, Thomas RJ, Allison TG, et al. Association of bodyweight with total mortality and with cardiovascular events in coronary artery disease: A systematic review of cohort studies. The Lancet. 2006; 368(9536):666-78. [DOI:10.1016/ S0140-6736(06)69251-9]

[22] Khajavi D, Parsa Z. [The development of psychometric properties of "the elderly's activities of daily life scale" (Persian)]. Journal of Motor Learning and Movement. 2014; 6(1):91-108. [DOI:10.22059/ JMLM.2014.50192

[23] Bortolussi G, Bejko J, Gallo M, Comisso M, Carrozzini M, Guglielmi C, et al. Coronary artery bypass grafting in elderly patients: Insights from a comparative analysis of total arterial and conventional revascularization. Journal of Cardiovascular Translational Research. 2016; 9(3):223-9. [DOI:10.1007/s12265-016-9688-y] [PMID]

[24] Chiariello L, Nardi P, Pellegrino A, Saitto G, Chiariello GA, Russo M, et al. Simultaneous carotid artery stenting and heart surgery: Expanded experience of hybrid surgical procedures. The Annals of Thoracic Surgery. 2015; 99(4):1291-7. [DOI:10.1016/j.athoracsur.2014.11.043] [PMID]

[25] Stamou SC, Nussbaum M, Stiegel RM, Reames MK, Skipper ER, Robicsek $F$, et al. Effect of body mass index on outcomes after cardiac surgery: Is there an obesity paradox? The Annals of Thoracic Surgery. 2011 91(1):42-7. [DOI:10.1016/j.athoracsur.2010.08.047] [PMID]

[26] Lehmkuhl E, Kendel F, Gelbrich G, Dunkel A, Oertelt-Prigione S, Babitsch B, et al. Gender-specific predictors of early mortality after coronary artery bypass graft surgery. Clinical Research in Cardiology. 2012; 101(9):745-51. [DOI:10.1007/s00392-012-0454-0] [PMID]

[27] Najafi M, Sheikhvatan M, Montazeri A. Quality of life-associated factors among patients undergoing coronary artery bypass surgery as measured using the WHOQOL-BREF: cardiovascular topic. Cardiovascular Journal of Africa. 2009; 20(5):284-9. [PMID] [PMCID] 
[28] Hosseini SR, Zabihi A, Jafariyan Amiri SR, Bijani A. [The relationship between chronic diseases and disability in daily activities and instrumental activities of dialy living in the elderly (English- Persian)]. Journal of Babol University of Medical Sciences. 2018; 20(5):23-9. http://jbums.org/ article-1-7302-en.html

[29] Udesh R, Mehta A, Gleason T, Thirumala PD. Carotid artery disease and perioperative stroke risk after surgical aortic valve replacement: $A$ nationwide inpatient sample analysis. Journal of Clinical Neuroscience. 2017; 42:91-6. [DOI:10.1016/j.jocn.2017.04.006] [PMID]

[30] da Rosa MP, Schwendler R, Lopes R, Portal VL. Carotid artery stenosis associated with increased mortality in patients who underwent coronary artery bypass grafting: a single center experience. The Open Cardiovascular Medicine Journal. 2013; 7:76-81. [DOI:10.2174/187419240 1307010076] [PMID] [PMCID] 